\title{
BULLE'IIN OF THE
}

\section{AMERICAN MATHEMATICAL SOCIETY}

\section{THE SEVENTH SUMMER MEETING OF THE AMERICAN MATHEMATICAL SOCIETY.}

The Seventh Summer Meeting of the American Mathematrcal Society was held at Columbia University, New York City, beginning on the afternoon of Wednesday, June 27, 1900, and extending through the two following days. As usual, the meeting was held in connection with that of the American association for the advancement of science, the Society being one of sixteen scientific bodies which have responded to the invitation of the Association to meet simultaneously with it. A considerable mutual advantage naturally arises from the arrangement. Attendance is facilitated and increased; broader interests are suggested; and a sense of scientific solidarity is experienced. The affiliated societies lose nothing of their individuality or independence, their relation to the Association not necessarily involving any more than mere simultaneous meeting. Plans for a closer official connection are, however, under consideration ; it may be found advisable to give the societies, as such, a representation in the councils of the Association. Such a federation would undoubtedly be beneficial in many respects. Meanwhile, a cordial spirit of coöperation prevails between the societies and the sections of the Association. The Mathematical Society devoted the two sessions of Thursday to a joint meeting with Section A, and the other sessions of the two bodies were arranged for mutual convenience of attendance. The Society contributed to the officers of the Association their common president, Professor R. S. Woodward, and to Section A the vice-president, Professor Asaph Hall, Jr., the secretary, Dr. W. M. Strong, and the vice-president elect, Professor James McMahon.

The early date of the meeting conflicted to some extent with the academic duties of many members, and reduced the period of preparation and accumulation of ma- 
terial from four to two months. Nevertheless, the occasion was in all respects one of the most successful in the history of the Society. The attendance reached the highest point previously recorded, the following fifty-six members having been registered as present:

Dr. W. G. Bullard, Dr. A. S. Chessin, Professor F. N. Cole, Dr. J. V. Collins, Professor L. L. Conant, Professor E. S. Crawley, Professor E. W. Davis, Dr. W. S. Dennett, Professor L. E. Dickson, Professor W. P. Durfee, Dr. J. C. Fields, Professor H. B. Fine, Professor T. S. Fiske, Mr. W. B. Ford, Rev. J. G. Hagen, Miss Carrie Hammerslough, Professor James Harkness, Mr. H. E. Hawkes, Dr. A. A. Himowich, Professor E. W. Hyde, Dr. S. A. Joffe, Mr. C. J. Keyser, Professor P. A. Lambert, Dr. James Maclay, Professor W. H. Maltbie, Professor Mansfield Merriman, Professor W. H. Metzler, Dr. G. A. Miller, Professor E. H. Moore, Professor F. Morley, Dr. D. A. Murray, Professor A. B. Nelson, Professor Simon Newcomb, Professor H. B. Newson, Professor W. F. Osgood, Professor Anna H. Palmié, Mr. J. C. Pfister, Professor James Pierpont, Dr. M. B. Porter, Dr. E. D. Roe, Professor F. H. Safford, Professor 'T. J. J. See, Professor P. F. Smith, Dr. Virgil Snyder, Dr. F. H. Stecker, Professor Ormond Stone, Dr. W. M. Strong, Professor J. H. Tanner, Professor C. L. Thornburg, Miss Mary Underhill, Professor J. H. Van Amringe, Professor L. A. Wait, Professor H. S. White, Professor C. B. Williams, Professor R. S. Woodward, Professor J. W. A. Young.

Ex-President Simon Newcomb presided at the opening of the first session, on Wednesday afternoon, afterward yielding the chair to Vice-President E. H. Moore, relieving President R. S. Woodward, who was also president of the Association. Professor H. S. White, Professor E. W. Hyde, and the Secretary were also called to the chair during the later sessions. Professor Ormond Stone presided at the joint session with Section A on Thursday morning. The Council announced the election of the following persons to membership in the Society : Mr. J. L. Coolidge, Harvard University ; Professor Peter Field, Carthage College; Mr. F. A. Giffin, University of Colorado; Wm. J. Greenstreet, M.A., Stroud, England; Mr. L. L. Locke, Fredonia, Pa.; Professor J. E. Manchester, Vincennes University; Professor Wm. J. Vaughn, Vanderbilt University. Six applications for membership were reported. The Council decided to set apart the life membership fund, now amounting to $\$ 600$, as a special fund to be devoted to such purpose as may hereafter be designated. 
The following papers were read at this meeting :

(1) Dr. A. S. CHEssin: "On the motion of a top, taking into account the rotation of the earth."

(2) Professor F. Morley: "On a mechanism for drawing trochoidal and allied curves."

(3) Mr. H. W. KUHN : "Theorems on imprimitive groups" (preliminary communication).

(4) Dr. H. E. Timerding: "Some remarks on tetrahedral geometry."'

(5) Professor H. B. Newson : "On singular transformations."

(6) Dr. Virgil SNyder : "On a special form of annular surfaces."

(7) Professor F. MoRLey : "On the rational quartic curve in space."

(8) Professor Paul Gondan: "Die Hesse'sche und die Cayley'sche Curve."

(9) Mr. H. E. HAwkes: "On hyper-complex number systems."

(10) Professor Maxime Bôcher : "Application of a method of d'Alembert to the proof of Sturm's theorem of comparison."

(11) Miss I. M. Schottenfels : "On groups of order $8 ! / 2 . "$

(12) Professor P. F. Sмiтh "On surfaces sibi-reciprocal under those contact transformations which transform spheres into spheres."

(13) Professor E. H. Moore : "A simple proof of the fundamental Cauchy-Goursat theorem."

(14) Professor W. F. Osgood : "On the existence of the Green's function for the most general simply connected plane region."

(15) Dr. J. V. CoLurns: "Quaternions and spherical trigonometry."

(16) Professor J. McMaнon : "Kelvin's treatment of instantaneous and permanent sources extended to certain cases in which a source is in motion."

(17) Dr. F. R. Moulton : "Oscillating satellites."

(18) Miss B. E. Grow : "The reduction of binary quantics to canonical forms by linear transformation."

(19) Dr. M. B. Porter: "Note on geometry on the nonsingular cubic."

Mr. Kuhn's paper was presented to the Society through Dr. G. A. Miller, Dr. Timerding's and Professor Gordan's through Professor H. S. White, Miss Schottenfels's through the Secretary, and Miss Grow's through Professor H. B. 
Newson. In the absence of the authors, Mr. Kuhn's paper was read by Dr. Miller, Dr. Timerding's and Professor Gordan's by Professor White, Professor McMahon's by Dr. Snyder, Dr. Moulton's by Professor Moore, and Miss Grow's by Professor Newson. The papers of Professor Bôcher, Miss Schottenfels, and Dr. Porter were read by title. 'The papers of Dr. Timerding and Miss Schottenfels appeared in the Bulletin for July, 1900. Abstracts of the other papers are given below.

The Friday afternoon session was mainly devoted to a discussion of the following question :

What courses in mathematics should be offered to the student who desires to devote one-half, one-third, or one-fourth of his undergraduate time to preparation for graduate work in mathematics?

The discussion was opened by the following papers :

Professor E. H. Moore: "Certain fundamental ideas which should be emphasized throughout the undergraduate course."

Professor J. Harkness: "The importance of some preliminary training in applied mathematics"; "Courses in differential calculus and differential equations."

Professor W. F. Osgood : "Shall we try to place the calculus as early as possible?" "How early in the course may the lecture method be used with profit?" "Are the best results in graduate work secured from students who have devoted most of their undergraduate time to mathematics or from those who have combined a fair amount of mathematics with a more general culture?"

Professor F. MorLey: "The theory of equations ; spherical trigonometry ; geometric conics."

Professor J. W. A. Young : "Collegiate preparation for the teaching of mathematics in secondary schools."

A separate report of the discussion, prepared by Professor W. H. Maltbie, is printed in the present number of the Bulletin, pages 14-24.

Professor Chessin's paper is an application of the general theory of relative motion as presented by the author in his paper "On relative motion" in the Transactions, volume 1, number 2, pages 116-169. The top here considered is a body of revolution with one point of its axis fixed on the surface of the earth. The problem of this so called Foucault top has not heretofore been solved and, indeed, it was thought that it did not lend itself to a solution. (See the memoir "On the application of Lagrange's method to 
problems of relative motion," by $\mathrm{Ph}$. Gilbert.) The motion of the earth is considered as a perturbation of that motion which the top would have if the earth did not rotate. Among the effects produced by the rotation of the earth may here be mentioned more especially that which appears in the precessional motion of the top. The precession may be expressed by the formula

$$
\frac{a}{N}-C \omega
$$

where $N$ and $\omega$ denote, respectively, the number of revolutions of the top per time unit and the angular velocity of rotation of the earth ; $a$ and $C$ are certain coefficients which depend on the initial deviation of the top from a vertical position, on the moments of inertia, etc. The sense of the revolutions of the top being fixed, say from left to right for an observer along the axis of the top with his feet at the fixed point of the axis, the precessional motion of the top may take place from left to right, or on the contrary from right to left, or indeed there may be no precession at all, according as $N a$ is greater than, less than, or equal to $\alpha: C$. These results are in harmony with similar conclusions reached by the author in the case of Foucault's pendulum, previously discussed.

The model shown by Professor Morley was an embodiment of the following idea : A rod $O A$ is pivoted at $O$ and a $\operatorname{rod} A P$ is hinged at $A$ to the former rod. When such a system moves in a plane, the rods having constant or proportional angular velocities, then $P$ describes the general trochoid. It is seen at once from the triangle of velocities that the normal at $P$ meets $O A$ at a point $N$ fixed in $O A$. Conversely, then, if a rod $P N$ passes through a point of $O A$, and has a toothed wheel at $P$, whose axis is $P N$, the wheel will describe a trochoid on a drawing-board. By adjustments the lengths $O A$ and $N A$ can be altered, so that the one apparatus traces all trochoids within a certain range; though not all possible trochoids. Further, by adjusting the angle which the axis of the wheel makes with $P N$, we can draw the curve which cuts at a given angle all the ring of trochoids obtained by rotating a trochoid about its center.

The groups which can be represented as imprimitive groups only when the degree equals the order are divided by Mr. Kuhn into those that can be represented transitively only in the regular form and those that can also 
SEVENTH SUMMER MEETING OF THE SOCIETY. [Oct.,

be represented as primitive groups which are not regular. The latter are shown to be the non-cyclical groups of order $p q$ ( $p$ and $q$ being different prime numbers). The former include $(a)$ the abelian groups; $(b)$ the Hamiltonian groups; and (c) those groups that contain non-invariant subgroups, each of which includes an invariant subgroup. The last are omitted by Dyck, Mathematische Annalen, volume 22. The paper includes several theorems relating to the construction of imprimitive groups whose degree is the product of two prime numbers and which are isomorphic to any transitive subgroup of the metacyclic group whose degree is one of the primes. By means of these theorems most of the imprimitive groups of degree fifteen, for example, can be written down at once.

Singular transformations in certain projective groups of the plane have been determined by Study, Taber, Rettger, and others. The object of Professor Newson's paper is to determine the character and type of these singular transformations. He finds in the case of the special linear homogeneous group in the plane, Study's case, that the singular transformations are of type II., while the continuous subgroups are of types I. and V. The singular transformations are characterized by a variable $\alpha$ and a constant value of $k$, viz, $k=-1$. These values of the parameters do not satisfy the necessary and sufficient relation for a one parameter group of type II., viz, $k=a^{\alpha}$. (See Bulletin, volume 6, number 10, page 438.) These singular transformations are obtained by combining the single involution perspective transformation of the group with all those of type V. in the group.

In Dr. Snyder's paper the equations of annular surfaces having two spherical directrices are first derived by means of Lie's equations connecting line sphere space for both the cases in which the directrices are distinct and coincident. A particular case is next given showing that a non-reducible cone of order 6 exists, all of whose lines of curvature are of order 6. On transforming this surface into line space, making a linear transformation upon its points, and then putting the new scroll back into an annular surface, the latter will be of order 12, not of revolution, but will have plane lines of curvature of order 6 . More generally, by revolving any plane curve about any line in the plane, transforming the surface generated into a scroll, making a certain projection of the scroll, then putting the new scroll into sphere space 
an annular surface is obtained having plane lines of curvature whose order is half that of the surface-the latter not being of revolution. The scrolls are also of particular form ; their asymptotic lines are of order only half that of the general scroll of the same type.

Professor Morley's second paper is in abstract as follows : It is known that the bitangent planes of a rational space quartic $R_{4}$ touch a quadric, which contains the tangents of the curve at the flexes or points of contact of the four stationary planes. Any tangent plane of this quadric cuts $R_{4}$ at four points $U$, such that the Hessian points $H$ of $U$ also lie in a plane. This plane again touches the quadric. Any tetrad $U+\lambda H$ of $R_{4}$ lies in a plane which touches the quadric. All the planes form a pencil whose axis is a generator of the quadric, of the system to which the flex lines belong. Among the planes of the pencil there are two which cut $R_{4}$ in a self apolar tetrad, three which cut $R_{4}$ in apolar pairs of points and three bitangent planes. The one set of three planes is the cubicovariant of the other; the two planes form the common Hessian of the two sets of three planes.

Professor Gordan's paper, which will be published in the Transactions, is in brief an exhaustive discussion of the curves of order or class 3, which have for their Hessian or Cayleyan a given curve of the same order or class. The author shows that these number 3 in general, but in special cases 1 or $\infty$; for each case the types are determined explicitly.

The point of departure for Dr. Hawkes's paper was furnished by a consideration of certain criticisms of Benjamin Peirce's Memoir on linear associative algebra, by Study, Molien, Cayley, and others. The paper shows how Peirce's methods can be naturally extended so as to arrive very directly at an enumeration of all number systems in less than six units, which are inequivalent, non-reciprocal, irreducible, and possess moduli.

Of the many theorems contained in Sturm's famous memoir of 1836, the two Theorems of Comparison may be regarded as most fundamental. Professor Bôcher has shown on other occasions (cf. Bulletin, April 1898 and Dec. 1899, p. 100) how the methods which Sturm used for establishing these theorems can be thrown into rigorous form. In the present paper a simpler and more direct method of establishing these 
theorems is given along lines suggested by passages, to which Professor H. Burkhardt kindly called the author's attention, in one of d'Alembert's papers on the vibration of strings (Memoirs of the Berlin Academy, volume 70 (1763), p. 242). The fundamental idea involved is the reduction of the linear differential equations of the second order to Riccati's equations by the well known change of dependent variable.

The problem discussed by Professor Smith is a generalization of the question treated by Moutard in 1864, viz., to find properties of surfaces enveloped by spheres intersecting a fixed sphere at right angles. The generalization is this : The surfaces to be studied are enveloped by spheres intersecting a fixed sphere at any constant angle. As distinguished from the problem discussed by Moutard, the question is one of the higher spherical geometry, in that the algebraic sign of the radius of a sphere must be regarded. This amounts to distinguishing between the two sides of a surface, as given, e.g., by the two directions of the normal ; and this distinction is indicated by the term, "oriented surface." The treatment of the problem is purely geometrical, being based upon a certain simple contact transformation, here defined for the first time. Adopting Sophus Lie's terminology, "linear spherical complex," for the aggregate of $\infty^{3}$ oriented spheres intersecting a fixed fundamental oriented sphere under constant angle, this definition is as follows: Given an oriented sphere $S$, then all $\infty^{2}$ oriented spheres tangent to $S$ and belonging to a spherical complex $A$ are tangent to a second oriented sphere $S^{\prime}$, and $S$ is said to transform into $S^{\prime \prime}$ by inversion in the complex $A$. The transformation is involutory. This general inversion depends upon five essential parameters, and includes as particular cases inversion in a sphere, the parallel transformation, symmetry in a plane, and the transformation by reciprocal directions originally defined by Laguerre in 1881 . The $\infty^{3}$ points of space constitute a particular spherical complex $\Omega$, and inversion in $\Omega$ merely turns over an oriented plane.

The relation of this general inversion to the general sphere-sphere contact transformation of fifteen essential parameters is determined by the theorem : The latter transformation is compounded either of a general inversion, a spherical inversion and a displacement, or these three compounded with inversion in $\Omega$. Various theorems on the general inversion are established, e. g. (1) A general inversion $A$ is uniquely compounded of a parallel transformation 
$D$, a spherical inversion $I$ and the same parallel transformation $D$, i. e., $A=D I D$. (2) A general inversion $A$ is compounded in two ways of a general inversion $A_{0}$, the inversion in $\Omega$, and the same inversion $A_{0}$, or $A=A_{0} \Omega A_{0}$. The last theorem leads to a representation of the $\infty^{3}$ oriented spheres of the complex $A$ by the $\infty^{3}$ points of space, in that the former transform into the latter by inversion in $A_{0}$. This representation is fundamental for the point of view adopted in the paper. The following result is now established :

A surface $F$ enveloped by $\infty^{2}\left(\infty^{1}\right)$ spheres belonging to the spherical complex $A$ is transformed by $A_{0}$ into a nonoriented primitive surface (curve) $f(c)$, regarded as the locus of its points. The order and class of $F$ are equal, and its entire section with the plane at infinity is along the imaginary circle. The centers of these spheres of $A$ lie on a surface $F_{0}$ (curve $C_{0}$ ), which is the transformed of $f(c)$ by a birational transformation whose fundamental curve is the imaginary circle. The characteristics of $F, F$, and $C_{0}$ are easily determined from those of $f$ and $c$. The intersection of the fundamental sphere of $A$ and the primitive is a double line (point) on $F$, while the remaining intersection of $F$ and the fundamental sphere is a line of curvature on $F$.

Particular interest attaches to the case where the primitive is a general cyclide without nodes. Then $F$ is the socalled surface of singularities of the general quadratic spherical complex, and the following results are established:

The surface of singularities of the general quadratic spherical complex is of order and class 24 , and contains the imaginary circle at infinity twelve times. It is sibi-reciprocal under six commutative general inversions $A_{i}$, with each of which is associated a cyclide $f_{i}$, from which as primitive the surface may be derived. The surface contains six double lines, sphero-quartics, the intersections of the $f_{i}$ and the fundamental spheres, and is the envelope in six ways of $\infty^{2}$ spheres belonging to an $A_{i}$ whose centers lie upon a quartic surface $F_{i}$ containing a double conic. The six $F_{i}$ are confocal, being inscribed in the minimum developable of order 24 circumscribing $F$, which is also the singular minimum developable of that surface. Thus the focal lines of the $F_{i}$ are the focal lines of $F$. The surface $F$ is enveloped by six systems of bitangent planes each belonging to a developable of order 8 and circumscribed to a sphere concentric with the corresponding fundamental sphere along a sphero-quartic. The lines of curvature of $F$ are algebraic, being the transformed of the lines of curvature of the prim- 
itive cyclides $f_{i}$ by the six inversions which transform the $f_{i}$ into $F$.

When the primitive is a circle (or straight line), the surface $F$ is the cyclide of Dupin. This leads to a very simple derivation of the properties of this surface. Some special cases departing from the general results are discussed. The problem is taken up also for the plane, the case in which the primitive is a bicircular quartic giving results analogous to that just given for the cyclide, the order and class being 16 and each circular point octuple.

It is pointed out that in papers on subjects related to that under discussion the method adopted has been that of deriving results from known theorems in line geometry by the line-sphere transformation of Sophus Lie. To this there are two objections: (1) Spherical geometry is essentially metric ; and those theorems in line geometry which lead by this correspondence to such results as are developed above are not a priori evident. (2) The correspondence fails except for the case of three dimensions. The discussion of this paper, operating entirely in the realm of spherical geometry, gives results which may be immediately generalized for $n$ dimensions. Thus, e.g., the relation of the problem treated in the paper to the minimum projection of points in a space of $n+1$ dimensions into the oriented spheres of space of $n$ dimensions is determined.

Goursat's memoirs [Acta $\dot{M a t h e m a t i c a, ~ v o l u m e ~} 4$ (1884); Transactions, volume 1 (1900)] in proof of Cauchy's integral theorem

$$
\int_{c} f(z) d z=0
$$

without assumption as to the continuity of the derivative $f^{\prime}(z)$ on the closed region bounded by the curve $C$ of integration lay deeper foundations for the Cauchy-Riemann theory of functions of a complex variable. One finds an abstract of these memoirs in the Bulletin for June, 1899, pp. 427-429. Goursat sets out by a direct process to evaluate the integral in question. In Professor Moore's paper, which will appear in the Transactions, the integral is by an indirect process proved to have the value zero. The essential elernents of the proof are those of Goursat's first paper ; by the modification indicated one avoids the necessity of using the lemma to the proof of which Goursat's second paper is devoted.

The following is a summary of Professor Osgood's paper, 
which appeared in full in the July number of the Transactions: The problem of mapping the interior of a simply connected plane region $T$ conformably on the interior of a circle depends for its solution on the proof of the existence of the Green's function corresponding to $T$. Hitherto the existence of this function has been established for regions $T$ bounded by a finite number of pieces of analytic curves, and, more generally, for regions bounded by a finite number of pieces of regular curves, $i$. e., curves whose tangent turns continuously as a moving point $p$ traces out the curve. That the boundary of a general simply connected region $T$ may be of an essentially more complicated character is shown by the following example: On the axis of abscissas assume at pleasure a set of points perfect but not dense throughout any interval whatsoever. Erect at each point of this set a perpendicular of unit length, drawn in the direction of the positive axis of ordinates. The region $T$ consists then of the points of the upper half plane that remain after these perpendiculars have been cut out. Thus the boundary of $T$ will in this case have a positive content if the content of the assumed set of points on the axis of abscissas was positive. Moreover, there now exist points of the boundary that cannot be approached along a continuous curve, namely, any interior point of a perpendicular about which perpendiculars cluster from both sides.

In the present paper the existence of the Green's function for the most general simply connected region $T$ is established by methods due to Poincaré, and thus it appears that the interior of any such region whatsoever can be transformed conformably on the interior of a circle.

Dr. Collins deduced the fundamental formulæ of spherical trigonometry by means of quaternions, employing the sine-cosine, in place of the scalar-vector notation.

The conception of a moving source treated in Professor McMahon's paper was suggested by the following problem set by a physicist who wished to use the solution in connection with an experiment: A source of heat (such as a candle) starts at a given point of a very long straight wire, and is moved with uniform velocity along the wire ; it is required to find the temperature at any assigned point of the wire at any future time ; and especially to compare the temperature gradient in front of the candle with that behind the candle, when these gradients have become practically stationary relatively to the moving source. The methods 
of Fourier are not directly applicable, but a solution can be obtained. by using Kelvin's expression for the effect of an instantaneous source of heat placed at a given point of an infinite rod, and superposing the temperature effects due to a succession of such instantaneous sources placed at successive points of the line. The solutions for a finite rod with various terminal conditions are then given ; and the corresponding problems for an infinite plane and for a finite rectangle are solved. The results can be extended to three dimensions.

Lagrange found particular solutions of the problem of three bodies of two types: ( $a$ ) the three bodies lie always in a straight line; $(b)$ the three bodies are always at the vertices of an equilateral triangle. These solutions still exist when one of the bodies becomes infinitesimal, and when the two finite bodies move in circles. The problem is to find whether solutions exist in which the infinitesimal body describes closed curves in the vicinity of the points defined by the Lagrangian solutions. In Dr. Moulton's discussion use is made of the methods employed by Poincaré in his prize memoir in the Acta Mathematica, volume 13. The results obtained have points of contact with Darwin's memoir in the Acta Mathematica, volume 21. The principal results are the following: Let $1-\mu$ and $\mu$ represent the masses of the finite bodies. Let $\mu=\mu_{0}+\lambda$. There is a periodic solution for every $\mu_{0}$ in which the infinitesimal body moves in the plane of the motion of the finite bodies, in the vicinity of each one of the Lagrangian straight line solutions, and in which the coordinates are expansible as power series in $\lambda$, vanishing with $\lambda$, and with periodic coefficients, the periods depending upon $\mu_{0}$. Thus there is an infinite number of orbits vanishing with $\mu=\mu_{0}$. In the case of the equilateral triangular points in the plane there are two infinities of orbits with different periods vanishing with $\mu_{0}=\mu_{0}$, if $\mu_{0}<.3086$. In the vicinity of the equilateral triangular points in space of three dimensions there is an infinity of solutions, with periods differing from the other two, which vanish with $\mu=\mu_{0}$ for every value of $\mu_{0}$.

Miss Grow's paper is in abstract as follows: The transformation

$$
T: \quad \begin{aligned}
& z_{1}=\lambda_{1} z_{1}^{\prime}+\mu_{1} z_{2}^{\prime}, \\
& z_{2}=\lambda_{2}^{\prime} z_{1}^{\prime}+\mu_{2} z_{2}^{\prime},
\end{aligned}
$$


converts $f=e_{z}^{n}$ where the coefficients of $f$ are independent, into the form

$$
f^{\prime}\left(z^{\prime}\right)=e_{\lambda}^{n} z_{1}^{\prime n}+\left(\begin{array}{l}
n \\
1
\end{array}\right) e_{\lambda}{ }^{n-1} e_{\mu} z_{1}{ }^{n-1} z_{2}{ }^{\prime}+\cdots+e_{\mu}{ }^{n} z_{2}{ }^{\prime n} .
$$

If it is possible to transform the quantic by $T$ so that any two assigned coefficients, for example the $(n-r+1)$ th and $(k+1)$ th, shall vanish simultaneously, the necessary and sufficient condition is

$$
\begin{aligned}
e_{\lambda}{ }^{r} e_{\mu}{ }^{n-r} & =0, \quad e_{\lambda}{ }^{n-k} e_{\mu}{ }^{k}=0 \\
(r=1,2, \cdots, \quad n-1 ; k & =n-1, \quad n-2, \cdots, 1 ; k+n-r) .
\end{aligned}
$$
Consider
(1) $e_{z}^{r} e_{\mu}^{n-r}$,
(2) $e_{z}^{n-k} e_{\mu}^{k}$
(3) $e_{z}^{n-r} e_{\lambda}^{r}$,
(4) $e_{z}^{k} e_{\lambda}^{n-k}$.

If (1) and (2) vanish simultaneously, their resultant $R_{n-r, k}$ vanishes; also if (3) and (4) vanish simultaneously, their resultant $R_{r, n-k}$ vanishes. $R_{n-r, k}$ and $R_{r, n-k}$ each have $[(n-r)(n-k)+r k]$ zeros in the plane; therefore (1) and (2) will vanish simultaneously when $\mu$ is a root of $R_{n-r, k}$, and (3) and (4) when $\lambda$ is a root of $R_{r, n-k} \cdot f$ is always a factor of $R_{n-r, k}$ and $R_{r, n-l k}$, or $R_{n-r, k}=f C_{n-r, k}$, and $R_{r, n-k}$ $=f C_{r, n-k}$.

The $z$-points of (1) and (2) are the $\lambda$-points of $R_{r, n-k}$. Similarly the $z$-points of (3) and (4) are the $\mu$-points of $R_{n-r, k}, i$. e., the $\mu$-points of $R_{n-r, k}$ and $\lambda$-points of $R_{r, n-k}$ are so related that

$$
e_{\lambda}^{n-r} e_{\mu}^{r}=0, \quad e_{\lambda}^{k} e_{\mu}^{n-k}=0 .
$$

When $r=k, C_{n-r, k}$ and $C_{r, n-k}$ coincide in $\bar{C}_{r}$. The roots of $\overline{C_{r}}$ go in pairs which have reciprocal relations. In the transformation $T$, let $\lambda$ and $\mu$ be roots of $C_{r, n-k}$ and $C_{n-r, k}$ respectively and call this set of transformations $T_{n-r, k}$. Interchange $\lambda$ and $\mu$ and call this set $T_{r, n-k}$. The nonsingular binary quantic can be linearly transformed by the $[(n-r)(n-k)+k r-n]$ transformations $T_{n-r, k}$ so that its $(n-r+1)$ th and $(k+1)$ th terms shall vanish, and by $T_{r, n-k}$ so that its $(n-k+1)$ th and $(r+1)$ th terms shall vanish. Also if $\lambda$ and $\mu$ are zeros of $\overline{C_{r}}$ in corresponding pairs, we get two sets of transformations $\bar{T}_{r}$ and $\bar{T}_{r}^{\prime}$ by means of which its $(r+1)$ th and $(n-r+1)$ th terms can be made to vanish in $2 r(r-n)+n(n-1)$ different ways.

The theory is applied to the cubic, quartic, quintic, and sextic. 
The following is a summary of Dr. Porter's paper : Consider $3 m-s-1$ arbitrary fixed points $P$ on a non-singular cubic $C_{3}$, and $u_{i}=\int_{a b}^{x_{i} y_{i}} d u$ the integral of first kind on $C_{8}$, (ab) being a point of inflexion. If an $m$-ic have a $s-1$ order contact at $u_{1}$, it will cut $C_{3}$ again at $u_{2}, s u_{1}+u_{2} \equiv C$ (mod. $\left.\omega, \omega^{\prime}\right)$ where $C=\Sigma u_{i}$ at the points $P:$ The Schliessungsproblem thus suggested yields at once a proof of Fermat's theorem $a^{n}-a \equiv 0$ (mod. $n$ (prime)) and the generalized form of the theorem $F(a, n) \equiv 0$ (mod. $n)$. When $m=1, s=2$, we have systems of closed polygons. In case the polygon is a triangle, the equation of $C_{3}$ referred to it may be written

$$
\frac{x}{y}+\frac{y}{z}+\frac{z}{x}+2 \eta=0 .
$$

The twenty-four in-circumscribed triangles thus determined fall into four groups, each associated with an inflexion triangle, and each triangle of a group six ways perspective with its associate inflexion triangle. This configuration of inflexion triangles and in-circumscribed triangles presents numerous interesting geometrical properties.

Columbia UnIVERsity.

F. N. Cole.

\section{THE UNDERGRADUATE MATHEMATICAL CURRICULUM.}

REPORT OF THE DISCUSSION AT THE SEVENTH SUMMER MEETING OF THE AMERICAN MATHEMATICAL SOCIETY.

The final session of the Seventh Summer Meeting of the Society was devoted to an organized discussion of the following question :

What courses in mathematics shall be offered to the student who desires to devote one-half, one-third, or one-fourth of his undergraduate time to preparation for graduate work in mathematics?

The following topics were also suggested as a general basis of discussion :

How early in the course may the lecture method be used with profit? 